Research Article

\title{
Adaptive Configuration Method of Low-Frequency Electromechanical Sampling Information in Building Electrical System
}

\author{
Bai Yu $(\mathbb{D})$ and Zhixin Zhang \\ College of Information, Tianjin University of Commerce, Tianjin 300134, China \\ Correspondence should be addressed to Bai Yu; yubai2020@21cn.com
}

Received 3 January 2021; Revised 9 April 2021; Accepted 6 May 2021; Published 20 May 2021

Academic Editor: Hussein Abulkasim

Copyright (c) 2021 Bai Yu and Zhixin Zhang. This is an open access article distributed under the Creative Commons Attribution License, which permits unrestricted use, distribution, and reproduction in any medium, provided the original work is properly cited.

\begin{abstract}
In order to enhance the reliability of the electrical systems in low frequency, an adaptive configuration method of low-frequency electromechanical sampling information based on thyristor controlled series compensation (TCSC) is designed. The electrical system is simplified to a linear invariant system, and a stochastic subspace identification (SSI) method is established by using the singular value decomposition principle to collect low-frequency electromechanical sampling information. The reference channel technology is introduced to design the reference channel covariance matrix to judge whether low-frequency information is generated and improve the efficiency of sampling information acquisition. The architecture and working principle of the controllable series compensation device are analyzed, and the test signal method is used to evaluate the low-frequency modes and the information required by the device among the electrical system regions of buildings. The alternative input signals are selected by comparing different input signal residue ratios. The TCSC device parameters are adjusted by the compensation residue phase method, so as to realize the adaptive configuration of different low-frequency electromechanical sampling information and ensure the stable operation of the electrical system. The experimental results show that the proposed configuration scheme can effectively improve the damping ratio of the system and has an excellent effect on suppressing the continuous oscillation under a lowfrequency fault.
\end{abstract}

\section{Introduction}

The building electrical system includes key content such as building power distribution, indoor lighting, outdoor landscape lighting, and management automation [1]. With the improvement of building quality, the structure of the building electrical system has become increasingly complex, and the interconnection between different areas has gradually become dense. Sampling in low-frequency electromechanical systems can obtain the electromechanical oscillation conditions based on the sampled information. The oscillation with a frequency range of $0.2 \sim 3 \mathrm{~Hz}$ belongs to low-frequency oscillation [2, 3]. During the oscillation process, the rotors of the generators participating in the oscillation will swing relatively, the power transmission of the transmission line will fail, and the continuous increase of the oscillation amplitude will affect the normal operation of the electrical system, even causing the system to disassemble.

For the problem of low-frequency electromechanical oscillation, Yaqi and Zhigang [4] proposed a predictive current control method using a traction grid-side converter model based on a continuous control set in the coordinate system. By discretizing the state equation of the AC side, the mathematical prediction model of the single traction gridside converter was derived. The performance function was solved, that is, the difference between the predicted current value and the reference value and the change in the control voltage, to calculate the optimal control variable. The bipolar sinusoidal pulse width modulation was fused, to solve the phenomenon of traction blockage caused by low-frequency oscillation. But the method could not extract effective control parameters. Based on the discrete Fourier transform 
adaptive notch filter, Shen et al. [5] reduced the harm of continuous vibration. The notch filter instead of the low-pass filter was used to eliminate the deviation of resonance frequency and oscillation frequency, which greatly simplified the suppression process and reduced the frequency of oscillation frequency detection. At the same time, an oscillation detection method was designed, where interval detection and prediction methods were used to shorten the detection time. However, the running time of the notch filter was too long to satisfy the immediacy of low-frequency electromechanical oscillation suppression in the electrical system.

In this research, a TCSC-based adaptive configuration method of low-frequency electromechanical sampling information is proposed. The linear invariant difference equation of the building electrical system is established. Under the premise of the singular value decomposition principle, a low-frequency electromechanical sampling information extraction method based on the improved SSI method is designed to determine whether the low-frequency electromechanical oscillation phenomenon exists. The TCSC device is used to complete the damping control, and the oscillation suppression mode is adaptively adjusted according to different oscillation levels.

\section{Low-Frequency Electromechanical Sampling Information Extraction Based on Improved SSI Method}

The building electrical system is a large-scale nonlinear system. When exploring system modes and modals, the system is generally simplified to a linear invariant system. The system measurement signals are obtained through time sequence, and the system is described as a set of linear invariant difference equations as shown in equation (1) [6], and the system also meets the convergence condition in equation (2).

$$
\begin{array}{r}
\left\{\begin{array}{r}
x(k T+T)=A x(k T)+B u(k T)+w(k T), \\
y(k T)=C x(k T)+D u(k T)+v(k T),
\end{array}\right. \\
E\left[\left(\begin{array}{c}
w(p T) \\
v(q T)
\end{array}\right)\left(w^{\prime}(p T) v^{\prime}(q T)\right)\right]=\left[\begin{array}{cc}
Q & S \\
S^{\prime} & R
\end{array}\right] \delta_{p q},
\end{array}
$$

where $x \in R^{n \times 1}$ is the system state column vector, $y \in R^{l \times 1}$ is the system output column vector, $u \in R^{m \times 1}$ is the known system input control column vector, $A \in R^{n \times n}$ is the system state matrix, $B \in R^{n \times m}$ is the system control matrix, $C \in R^{l \times n}$ is the system output matrix, $D \in R^{l \times m}$ is the system control output matrix, $T$ represents the time-step length, $k$, $p$, and $q$ are all time-step coding, $w$ is the system input stochastic interference column vector, and $v$ is the system output measurement stochastic deviation column vector.

In most states, the system control input $u$ is an unknown quantity. By including the influence of $u$ in the system stochastic interference and measurement stochastic deviation $[7,8]$, we get

$$
\left\{\begin{aligned}
x(k T+T) & =A x(k T)+w(k T), \\
y(k T) & =C x(k T)+v(k T) .
\end{aligned}\right.
$$

Regarding the state matrix $A$ of the differential model, eigenvalue and relative right eigenvector $\varphi_{i}$ are obtained by eigen analysis. The eigenvalues of the continuous system model can be obtained by

$$
s_{i}=\frac{\ln \left(e_{i}\right)}{T} .
$$

The modal corresponding to mode $s_{i}$ of each output channel in the output column vector $y$ is

$$
V_{i}=C \varphi_{i}
$$

The above calculation process can be summarized as follows. Under the action of interference input $w$ and measurement deviation $v$ of approximate white noise, the building electrical system obtains the time sequence of the output signal $y$ in the system through the measurement value and obtains the system state matrix and the output matrix through the relevant information extraction algorithm. The system mode and modal status can be specified according to equations (4) and (5), which in this paper refers to detecting whether the low-frequency electromechanical has oscillation phenomenon.

The SSI method is a commonly used method in the field of system information collection. It is built on the principle of singular value decomposition [9] and has strong numerical stability. Through measurement, the system output time sequence can be obtained as $\{y(0), y(T), \ldots, y((N-1) T), y(N T)\}$.

The output covariance matrix of the time lag $i T$ is

$$
\begin{aligned}
\Lambda_{i} & =E\left[(y(k+1) T) y^{\prime}(k T)\right] \\
& =\lim _{N \longrightarrow \infty} \frac{1}{N} \sum_{k=0}^{N-1}\left((y(k+1) T) y^{\prime}(k T)\right) .
\end{aligned}
$$

The following block Toeplitz matrix is set:

$$
T_{1 \mid i}=\left[\begin{array}{cccc}
\Lambda_{i} & \Lambda_{i-1} & \cdots & \Lambda_{1} \\
\Lambda_{i+1} & \Lambda_{i} & \cdots & \Lambda_{2} \\
\cdots & \cdots & \cdots & \cdots \\
\Lambda_{2 i-1} & \Lambda_{2 i-2} & \cdots & \Lambda_{i}
\end{array}\right] .
$$

In the real scene, the length of the sequence is limited [10], and the covariance matrix should be approximated by

$$
\Lambda_{i} \approx \widehat{\Lambda}_{i}=\frac{1}{N} \sum_{k=0}^{N-1}\left((y(k+1) T) y^{\prime}(k T)\right) .
$$

Then, the calculation process of the SSI algorithm is as follows. The time sequence of system output $y$ is obtained through measurement; the covariance matrix $\Lambda_{1}, \Lambda_{2}, \ldots, \Lambda_{i}$ under each time lag is calculated according to equation (8), where $i$ satisfies $l i \gg n$, and equation (7) is used to construct the Toeplitz matrix $T_{1 \mid i}$; singular value decomposition is performed on $T_{1 \mid i}$; then, we get 


$$
T_{1 \mid i}=U S V^{T}=\left[\begin{array}{ll}
U_{1} & U_{2}
\end{array}\right]\left[\begin{array}{cc}
S_{1} & 0 \\
0 & 0
\end{array}\right]\left[\begin{array}{c}
V_{1}^{T} \\
V_{2}^{T}
\end{array}\right]=U_{1} S_{1} V_{1}^{T} .
$$

The extended observability matrix is obtained:

$$
Q_{i}=U_{1} S_{1}^{1 / 2} Z \text {. }
$$

The output matrix and state matrix are obtained:

$$
\begin{aligned}
& C=Q_{i}(1: l,:), \\
& A=Q_{i}(1: l(i-1),:)^{\dagger} Q_{i}(l+1: l i,:) .
\end{aligned}
$$

In the SSI algorithm, in order to obtain the modal information of some busbars relative to a certain model in the building electrical system [11], it is necessary to clarify whether there is oscillation information inside the electromechanical system. With the continuous expansion of the system scale, the number of system buses will increase, and the measurement of the buses will be included in the output column vector. The reference channel technology is used to maximize operational efficiency without reducing the accuracy of sampling information extraction.

The concept of output channel originates from the field of signal processing. The concept of the output channel in this paper is the output signal measured by the system. The measurement output series obtained in modal analysis generally contains much redundant information [12, 13], and the amount of calculation can be reduced by reducing the redundant information. The reference channel is a subset $y^{\text {ref }} \in R^{r}$ of the output column vector, where $r$ is the dimension of the reference channel, and the following equation is obtained:

$$
y=\left[\begin{array}{c}
y^{\text {ref }} \\
y^{\sim \text { ref }}
\end{array}\right]
$$

where

$$
\begin{aligned}
y^{\text {ref }} & =L y, \\
L & =\left[\begin{array}{ll}
I_{r} & 0
\end{array}\right],
\end{aligned}
$$

where $y^{\sim \text { ref }}$ is the output vector except the reference channel in the initial vector [14], and the reference channel covariance matrix is recorded as

$$
\begin{aligned}
\Lambda_{i}^{\mathrm{ref}} & =E\left[(y(k+i) T)\left(y^{\mathrm{ref}}(k T)\right)^{\prime}\right] \\
& =\lim _{N \longrightarrow \infty} \frac{1}{N} \sum_{k=0}^{N-1}\left((y(k+1) T) y^{\mathrm{ref}}(k T)^{\prime}\right),
\end{aligned}
$$

where $\Lambda_{i}^{\text {ref }}$ is the output covariance matrix with the time lag of the reference channel being $i T$.

$\Lambda_{i}^{\text {ref }}$ is introduced into equation (7) to form a brand-new matrix $T_{1 \mid i}^{\mathrm{ref}}$, and the selection rule for setting iteration parameter $i$ is $r i \gg n$. After introducing the reference channel, the value of $\Lambda_{i}^{\text {ref }}$ is calculated, and singular value decomposition is performed. According to equation (5), the current low-frequency motor oscillation condition of all output channels can be calculated.

\section{Adaptive Configuration of Low-Frequency Electromechanical Sampling Information Based on TCSC}

Through the above content, it is possible to clarify whether there is oscillation information inside the low-frequency electromechanical system. If oscillation information is generated, TVSC can be used to adaptively configure different degrees of oscillation problems to ensure the stability of the building electrical system.

The TCSC device can adjust the reactance value of the series compensation device in a large area [15], which can enhance the power transmission quality of the high-intensity power transmission system, effectively control the power flow of the system, and reduce the voltage deviation. The topology of the device adopts a low-level control design, which can well reduce the generation of low-frequency electromechanical oscillation information.

Damping is the key to damping the low-frequency oscillation information of the device. The control process is presented in Figure 1.

The electrical system input has two parts of voltage and line transmission power. The damping output is formed through processes such as deviation comparison, dead zone control, and phase shifting rectification. $Z_{\mathrm{TCSC} 0}$ represents the command impedance issued by the personal computer, $\Delta Z_{1}$ is the damping impedance of power loop, $\Delta Z_{2}$ represents the damping impedance of voltage loop, and $Z_{\text {TCSC }}$ is the output impedance of final stage. The performance and parameters of each link are defined as follows.

$V$ is the positive sequence voltage monitoring value of reference point, $V_{0}$ is the voltage reference value of reference point, that is, the steady value before oscillation occurs, $P_{L 0}$ is the transmission power reference value of the transmission channel where the device is located, and $P_{L}$ is the power transmission monitoring value of the transmission channel. The deviation comparison has the characteristics of firstorder inertia [16], its time constant presents the characteristics of the real measurement device, and the transfer function of the deviation comparison link is $K_{m} /\left(1+T_{m} S\right)$.

The dead zone is to prevent the device from oscillating under a small stochastic interference and plays a certain protective role. DC blocking reset is a high-pass filter that can prevent the generation of DC components in the TCSC device's control input signal after low-frequency oscillations, thereby reducing its negative impact on the oscillation suppression effect. Phase shifting is the core content of damping control, allowing the capacitive reactance of the device to obtain the phase difference between the control input signal and the control input signal with the change of the control input signal [17]. If the appropriate phase difference is selected, the change in the capacitive reactance of TCSC will fit the change of the phase difference, which helps adaptively obtain an additional matrix close to the phase of the speed deviation and deal with the low-frequency oscillation of the building electrical system, with the transfer function being $\left(1+T_{1} S\right) /\left(1+T_{2} S\right)$. The transient stability control 
link is a control process that improves the transient stability of the electrical system. After the oscillation is eliminated, the equivalent reactance of the device is immediately adjusted to a fixed value for forced compensation to reduce the electrical distance between the transmitting and receiving ends. In the protection limit link, since the capacitive reactance of the TCSC can only be adjusted in a fixed range, the adjustment range depends on the performance of the capacitor bank, the overvoltage protection level, and the current through the TCSC. The protection limit link can take reasonable constraints on the command reactance obtained by the transient stability control and damping control [18], thereby guaranteeing the normal operation of the protection device.

In order to meet the diversified operation mode of the building electrical system, the input signal of the TCSC device should be selected reasonably to improve the robustness of the adaptive configuration. When the device is not connected, the test signal is used to obtain the open-loop transfer function from the anti-group reference signal to the input signal:

$$
G(s)=\frac{\Delta B(s)}{\Delta X_{0}(s)}=\sum_{i=1}^{n} \frac{R_{i}}{s-\lambda_{i}},
$$

where $\lambda_{i}$ is the pole of $G(s)$ and $R_{i}$ represents the residue relative to $\lambda_{i}$.

The transfer function clarifies the controllable and observable conditions in the electrical system. Therefore, the movement of the pole $\lambda_{i}$ in $G(s)$ in the signal $B$ is observable. Assuming $\lambda_{i}$ is the pole of the low-frequency oscillation information mode, its relative residue $R_{k}$ can be used as an observable indicator. Calculating $\left|R_{k}\right|$ can clarify the change of observable level with the change of different operating modes, and the residue ratio is used as the indicator to weigh the observability of the alternative input signal of the TCSC device [19]. The residue is expressed as

$$
\rho_{k}=\frac{\left|R_{k, B I}\right|}{\left|R_{k, B 0}\right|}
$$

where $k$ represents the low-frequency electromechanical regional oscillation information code and $\left|R_{k, B I}\right|$ and $\left|R_{k, B 0}\right|$ represent the digital modulus of the first regional oscillation information in the adaptive configuration operation mode and the digital modulus of the first regional oscillation information in the standard operation mode, respectively. In different operation modes, the less the residue ratio changes, the better the robustness of the input signal to the system operation.

TCSC device parameters can be adjusted using the compensation residue phase algorithm [20]. The device is expressed in the form of a transfer function:

$$
\mathrm{KH}(s)=\frac{K}{1+s T_{1}} \cdot \frac{s T_{w}}{1+s T_{w}} \cdot\left[\frac{1+s T_{2}}{1+s T_{3}}\right]^{m} .
$$

The adaptive configuration parameters of the phase shifting link are solved by

$$
\left\{\begin{array}{l}
\phi=180^{\circ}-\arg R_{k}, \\
\alpha=\frac{T_{3}}{T_{2}}=\frac{[1-\sin (\phi / m)]}{[1+\sin (\phi / m)]}, \\
T_{2}=\frac{1}{\left(\omega_{k} \sqrt{\alpha}\right)}, \\
T_{3}=\alpha T_{2} .
\end{array}\right.
$$

Now, the whole process of the adaptive configuration of low-frequency electromechanical sampling information is completed, which greatly reduces the probability of lowfrequency electromechanical failures and provides a solid foundation for the stable operation of the building electrical system.

\section{Simulation Experiment}

The power system analysis integrated program, i.e., Power System Analysis Software Package (PSASP), was used to study the damping characteristics of the building electrical system without a TCSC device. The operation mode was that A transmitted $1000 \mathrm{MW}$ active power to $\mathrm{B}$, and the transmission result had 4 dominant low-frequency oscillation modes, i.e., 3 inter-regional oscillations and 1 intra-regional oscillation, indicating the unstable state of the system.

In order to prove that the proposed method could effectively improve the damping of the building electrical system, a TCSC device was installed in the system, and a 6\% step interference signal was added to the excitation voltage amplitude of the system. Adaptive configuration optimization was conducted on the system's low-frequency electromechanical information and its circuit. The simulation results are shown in Figures 2 and 3. Table 1 shows the dataset showing comparison of low-frequency speed information of building electrical system. Table 2 shows the dataset showing comparison of low-frequency power information of link circuit.

As can be seen from Figures 2 and 3, the installed TCSC devices could effectively suppress the generation of lowfrequency information. This was because the proposed method provided sufficient damping for each low-frequency mode and alleviated the adverse effects caused by the oscillation of the low-frequency electromechanical system.

In order to verify the ability of the TCSC-based adaptive configuration method on suppressing low-frequency information in the state of large interference, three shortcircuit faults were set near the system's link circuit, and the line was disconnected after $0.2 \mathrm{~s}$; then, the output power of the system's line is shown in Figure 4.

It can be seen from Figure 4 that the system could maintain stable operation in the operation mode of a single link circuit before and after using the adaptive configuration device, but the TCSC device showed a better optimization effect. The advantage of the TCSC device was that it used the improved SSI method to determine whether the system had low-frequency information. As a result, the overall status of 


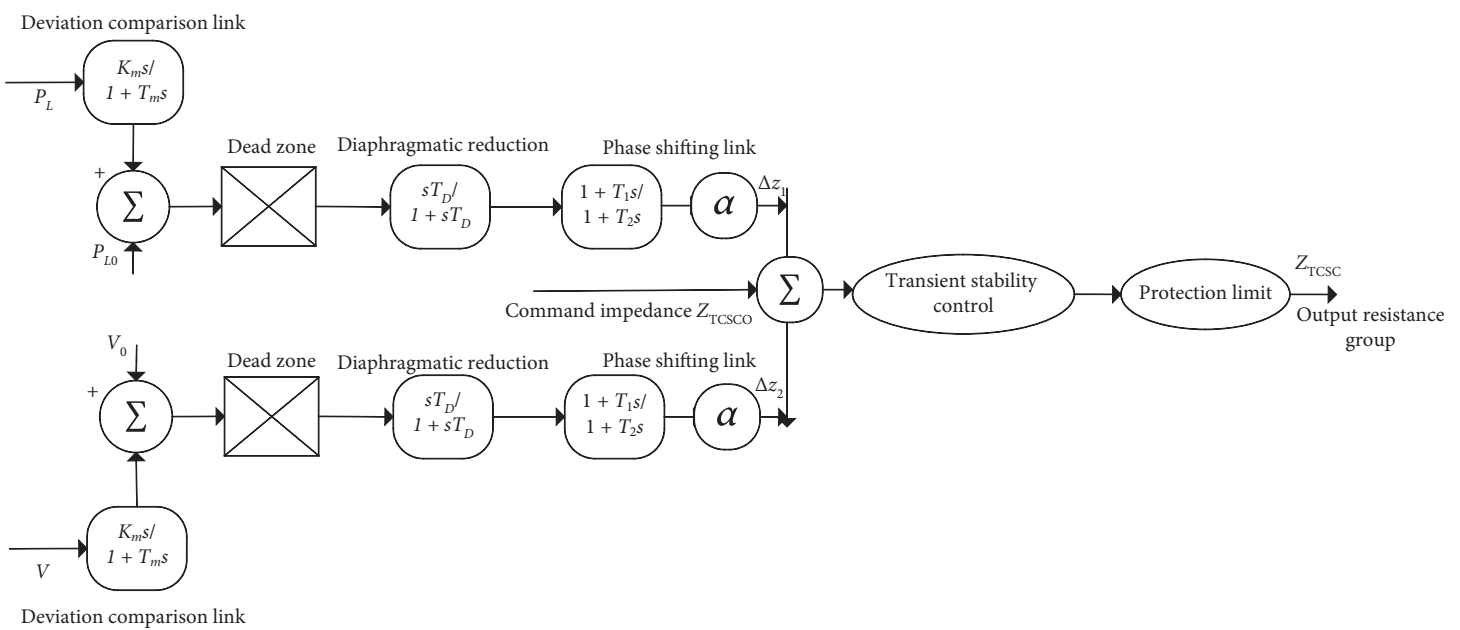

FIgURE 1: Schematic diagram of the damping control process.

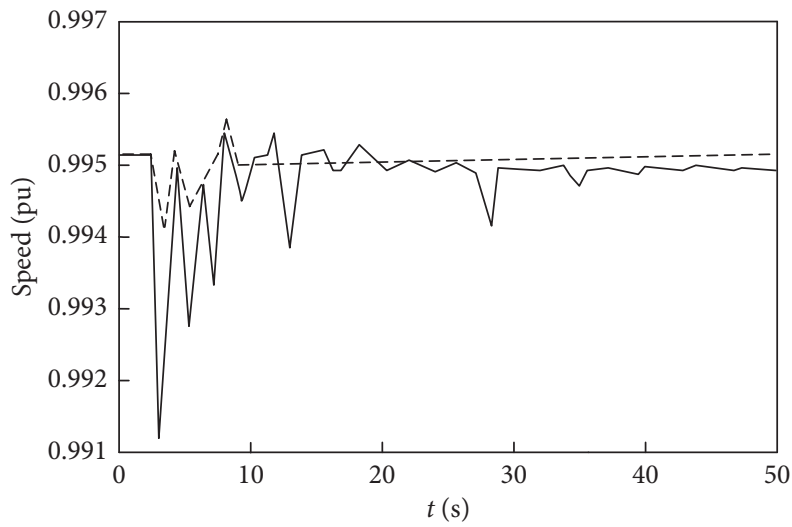

--- The method of this paper

— Not optimized

Figure 2: Comparison of low-frequency speed information of building electrical system.

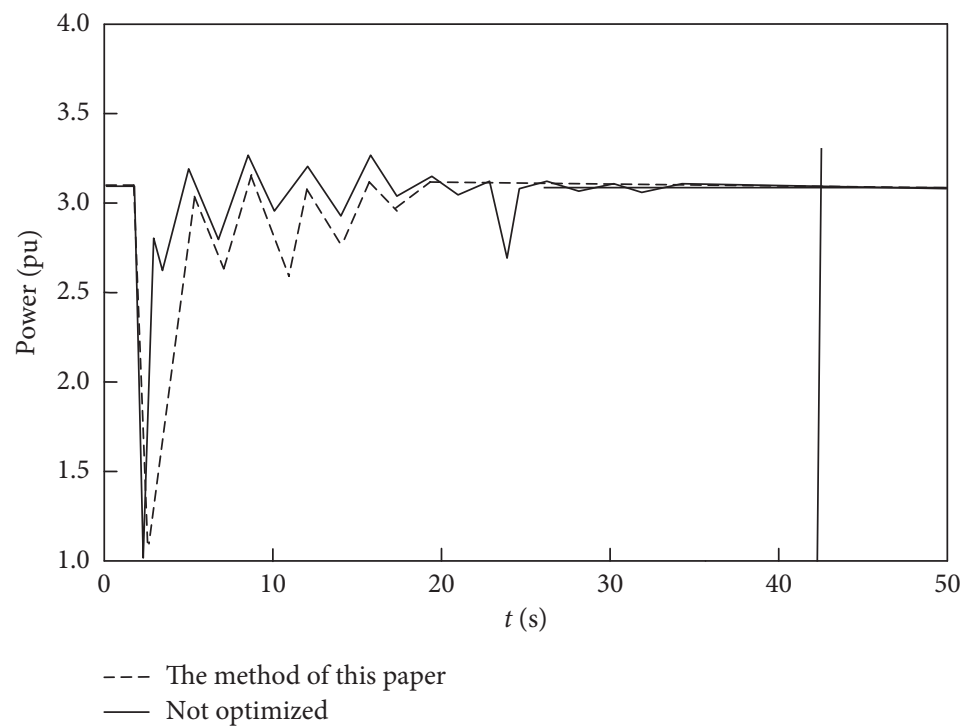

FIGURE 3: Comparison of low-frequency power information of link circuit. 
TABLE 1: Dataset showing comparison of low-frequency speed information of building electrical system.

\begin{tabular}{|c|c|c|c|}
\hline S.no. & Time (s) & $\begin{array}{l}\text { Not optimized } \\
\text { Speed }(\mathrm{pu})\end{array}$ & $\begin{array}{l}\text { Proposed method } \\
\text { Speed }(\mathrm{pu})\end{array}$ \\
\hline 1 & 0 & 0.9951 & 0.9951 \\
\hline 2 & 0.5 & 0.9951 & 0.9951 \\
\hline 3 & 1 & 0.9951 & 0.9951 \\
\hline 4 & 3 & 0.9912 & 0.9940 \\
\hline 5 & 3 & 0.9942 & 0.9940 \\
\hline 6 & 4 & 0.9951 & 0.9942 \\
\hline 7 & 5 & 0.9935 & 0.9945 \\
\hline 8 & 6 & 0.993 & 0.9946 \\
\hline 9 & 7 & 0.9948 & 0.9947 \\
\hline 10 & 8 & 0.9935 & 0.9949 \\
\hline 11 & 9 & 0.9955 & 0.9951 \\
\hline 12 & 10 & 0.994 & 0.9952 \\
\hline 13 & 11 & 0.9955 & 0.9952 \\
\hline 14 & 13 & 0.994 & 0.9952 \\
\hline 15 & 15 & 0.99508 & 0.9952 \\
\hline 16 & 16 & 0.9951 & 0.9952 \\
\hline 17 & 17 & 0.9949 & 0.9952 \\
\hline 18 & 19 & 0.9951 & 0.9952 \\
\hline 19 & 20 & 0.9949 & 0.9952 \\
\hline 20 & 22 & 0.995 & 0.9952 \\
\hline 21 & 25 & 0.9949 & 0.9952 \\
\hline 22 & 29 & 0.9942 & 0.9952 \\
\hline 23 & 30 & 0.9949 & 0.9952 \\
\hline 24 & 35 & 0.9948 & 0.9952 \\
\hline 25 & 40 & 0.9949 & 0.9952 \\
\hline 26 & 50 & 0.9949 & 0.9952 \\
\hline
\end{tabular}

TABLE 2: Dataset showing comparison of low-frequency power information of link circuit.

\begin{tabular}{|c|c|c|c|}
\hline S.no. & Time (s) & $\begin{array}{l}\text { Not optimized } \\
\text { Power }(\mathrm{pu})\end{array}$ & $\begin{array}{l}\text { Proposed method } \\
\text { Power }(\mathrm{pu})\end{array}$ \\
\hline 1 & 0 & 3.1 & 3.1 \\
\hline 2 & 0.5 & 3.1 & 3.1 \\
\hline 3 & 1 & 3.1 & 3.1 \\
\hline 4 & 2 & 1.1 & 1.1 \\
\hline 5 & 3 & 2.6 & 1.2 \\
\hline 6 & 4 & 2.8 & 2.2 \\
\hline 7 & 5 & 3.1 & 3.0 \\
\hline 8 & 6 & 2.9 & 2.6 \\
\hline 9 & 7 & 2.6 & 2.4 \\
\hline 10 & 8 & 2.7 & 2.4 \\
\hline 11 & 9 & 2.9 & 2.4 \\
\hline 12 & 10 & 3.2 & 3.1 \\
\hline 13 & 11 & 3.0 & 2.8 \\
\hline 14 & 13 & 3.1 & 3.0 \\
\hline 15 & 15 & 3.0 & 2.8 \\
\hline 16 & 16 & 3.2 & 3.0 \\
\hline 17 & 17 & 3.3 & 3.1 \\
\hline 18 & 19 & 3.0 & 2.9 \\
\hline 19 & 20 & 3.1 & 3.1 \\
\hline 20 & 22 & 3.1 & 3.0 \\
\hline 21 & 25 & 2.55 & 3.1 \\
\hline 22 & 29 & 3.0 & 3.1 \\
\hline 23 & 30 & 3.0 & 3.1 \\
\hline 24 & 35 & 3.1 & 3.1 \\
\hline 25 & 40 & 3.1 & 3.1 \\
\hline 26 & 50 & 3.1 & 3.1 \\
\hline
\end{tabular}




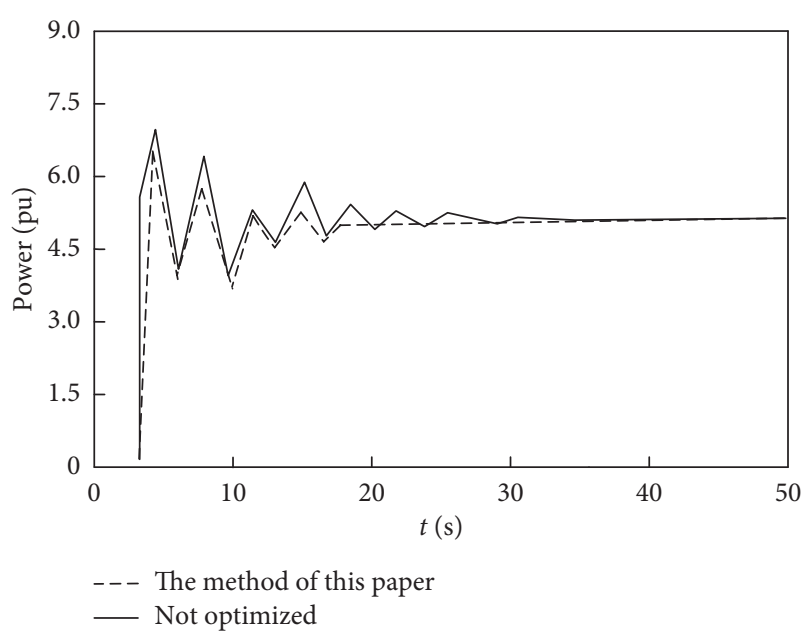

FIgURE 4: Comparison of low-frequency information of the line's power.

the system could be accurately analyzed, and different methods were adopted to optimize strategies according to the amount of low-frequency information and the degree of oscillation of the system, thereby obtaining a better operation effect.

\section{Conclusion}

In order to ensure the stability of the low-frequency electromechanical operation of the building electrical system, a TCSCbased adaptive configuration method of low-frequency electromechanical sampling information is designed in this research. The proposed method has simple calculation, effectively improves the damping of the building electrical system, and has a good effect on suppressing the low-frequency electromechanical oscillations of various degrees.

However, when measuring the output time sequence of the system, the stages of the system application were ignored, which might lead to poor accuracy of the time sequence value. This problem should be solved in further research.

\section{Nomenclature}

$\begin{array}{ll}T: & \text { The time-step length } \\ k, p, \text { and } q: & \text { Time-step coding } \\ w: & \text { The system input stochastic interference } \\ & \text { column vector } \\ v: & \text { The system output stochastic deviation } \\ & \text { column vector } \\ u: & \text { The system control input } \\ A: & \text { The state matrix } \\ e_{i}: & \text { Eigenvalue } \\ \varphi_{i}: & \text { Relative right eigenvector } \\ s_{i}: & \text { Continuous system model } \\ w: & \text { Interference input of white noise } \\ v: & \text { Measurement deviation of white noise } \\ y: & \text { Output signal } \\ \Lambda_{1}, \Lambda_{2}, \ldots, \Lambda_{i}: & \text { The covariance matrix } \\ T_{1 \mid i}: & \text { Toeplitz matrix }\end{array}$

$Q_{i}:$

C:

$r:$

$\Lambda_{i}^{\mathrm{ref}}$ :

$Z_{\mathrm{TCSC} 0}$ :

$\Delta Z_{1}$ :

$\Delta Z_{2}$ :

$Z_{\text {TCSC }}$ :

$V:$

$V_{0}$ :

$P_{L 0}$ :

$P_{L}$ :

$K_{m} /\left(1+T_{m} S\right)$ :

$G(s)$ :

$\lambda_{i}$ :

$R_{i}$ :

$\lambda_{i}$ :

$R_{k}$ :

$K H(s):$

$\left|R_{k, B I}\right|$ :

$\left|R_{k, B 0}\right|:$

$\rho_{k}$ :

$\varnothing, \alpha, T_{2}, T_{3}$ :

$V_{i}:$

\section{Data Availability}

The data used to support the findings of this study are available from the corresponding author upon reasonable request.

\section{Additional Points}

Highlights. (1) The main aim is to enhance the reliability of the electrical systems in low frequency. (2) Adaptive electromechanical sampling information based on TCSC is designed. (3) The architecture and working principle of the controllable series compensation device are analyzed. (4) The test signal method is used to evaluate the low-frequency modes. (5). The information is required by the device among the electrical system regions of buildings. (6) The alternative input signals are selected by comparing different input signal residue ratios. (7) The experimental results show that the scheme can effectively improve the damping ratio of the system. (8) The results show that the scheme suppresses the continuous oscillation under a low-frequency fault.

\section{Conflicts of Interest}

The authors declare that they have no conflicts of interest.

\section{Acknowledgments}

This study was supported by the Natural Science Foundation of Tianjing (no. 19JCTPJC53400). 


\section{References}

[1] M. Abdollahi, J. I. Candela, J. Rocabert, M. A. Elsaharty, and P. Rodriguez, "Novel analytical method for dynamic design of renewable SSG SPC unit to mitigate low-frequency electromechanical oscillations," IEEE Transactions on Power Electronics, vol. 35, no. 7, pp. 7532-7544, 2020.

[2] M. Yang, Y. Wang, C. Cai, Z. Liu, H. Zhu, and S. Zhou, "Monocular vision-based low-frequency vibration calibration method with correction of the guideway bending in a longstroke shaker," Optics Express, vol. 27, no. 11, p. 15968, 2019.

[3] W. Yang, P. Norrlund, J. Bladh, J. Yang, and U. Lundin, "Hydraulic damping mechanism of low frequency oscillations in power systems: quantitative analysis using a nonlinear model of hydropower plants," Applied Energy, vol. 212, pp. 1138-1152, 2018.

[4] W. Yaqi and L. Zhigang, "Suppression research regarding low-frequency oscillation in the vehicle-grid coupling system using model-based predictive current control," Energies, vol. 11, no. 7, p. 1803, 2018.

[5] H. Shen, J. Xu, and Y. Kang, "A fast adaptive resonance suppression scheme for high-damping system using R-SDFT detection," International journal of Electronics, vol. 106, no. 7-9, pp. 976-991, 2019.

[6] H. Shokouhandeh and M. Jazaeri, "An enhanced and autotuned power system stabilizer based on optimized interval type-2 fuzzy PID scheme," International Transactions on Electrical Energy Systems, vol. 28, no. 1, pp. 1-19, 2018.

[7] U. Agrawal, J. Follum, J. W. Pierre, and D. Duan, "Electromechanical mode estimation in the presence of periodic forced oscillations," IEEE Transactions on Power Systems, vol. 34, no. 2, pp. 1579-1588, 2019.

[8] S. G. Bortnikov, V. S. Aliev, and I. A. Badmaeva, "VO2 film temperature dynamics at low-frequency current self-oscillations," Journal of Applied Physics, vol. 123, no. 7, pp. 75701.1-75701.6, 2018.

[9] V. J. Boucher, A. C. Gilbert, and B. Jemel, "The role of lowfrequency neural oscillations in speech processing: revisiting delta entrainment," Journal of Cognitive Neuroscience, vol. 31, no. 8, pp. 1-11, 2019.

[10] P. Liu, S. Liu, H. Yang, X. Kang, and S. Pan, "K-band optoelectronic oscillator based on a double-Brillouin-frequency shifter," Optical Engineering, vol. 58, no. 10, p. 1, 2019.

[11] S. Qiang, W. Xinpeng, and W. Yixuan, "Oscillation suppression in the sense mode of a high-Q MEMS gyroscope using a simplified closed-loop control method," Sensors, vol. 18, no. 8, Article ID 2443, 2018.

[12] A. H. Naghshbandy and A. Faraji, "Coordinated design of PSS and unified power flow controller using the combination of CWT and Prony methods with the help of SPEA II multiobjective optimisation algorithm," IET Generation, Transmission \& Distribution, vol. 13, no. 21, pp. 4900-4909, 2019.

[13] F. Sun, J. Zou, and H. B. Chan, "In-situ control of electrical properties of nanoelectromechanical resonators by electromigration for self-sustained oscillations," Applied Physics Letters, vol. 115, no. 20, Article ID 203101, 2019.

[14] P. Wang, Z. He, K. R. Thompson, and J. Sheng, "Modulation of near-inertial oscillations by low-frequency current variations on the inner scotian shelf," Journal of Physical Oceanography, vol. 49, no. 2, pp. 329-352, 2019.

[15] A. M. S. Fransco and E. Echer, "Wavelet analysis of low frequency plasma oscillations in the magnetosheath of Mars," Advances in Space Research, vol. 65, no. 9, pp. 2090-2098,
2020, https://www.sciencedirect.com/science/article/abs/pii/ S027311771930657X.

[16] S. Ahmed, X. Zou, N. Jaber, M. I. Younis, and H. Fariborzi, “A low power micro-electromechanical resonator-based digital to analog converter," Journal of Microelectromechanical Systems, vol. 29, no. 3, pp. 320-328, 2020.

[17] Y. Dong, X. Zhang, and R. Yu, "Stability control and system protection scheme for power grid with hydropower pool and multi-DC weak sending-end," Dianli Xitong Zidonghua/Automation of Electric Power Systems, vol. 42, no. 22, pp. 19-25, 2018.

[18] A. Galetzka, Z. Bontinck, and U. Romer, "A multilevel Monte Carlo method for high-dimensional uncertainty quantification of low-frequency electromagnetic devices," IEEE Transactions on Magnetics, vol. 55, no. 8, pp. 1-12, 2019.

[19] R. Wu, P. C. E. Roberts, C. Soutis, and C. Diver, "Downrange manoeuvre and oscillation suppression of a self-regulating centrifugally deployed flexible heat shield using a controlled reaction wheel," Acta Astronautica, vol. 161, no. 2, pp. 415-424, 2019.

[20] S. W. Hwang, J.-H. Bak, J. Yoon, and J. H. Park, "Oscillation reduction and frequency analysis of under-constrained cabledriven parallel robot with three cables," Robotica, vol. 38, no. 3, pp. 375-395, 2020. 